\title{
Vortices with fractional flux in two-gap superconductors and in extended Faddeev model
}

\author{
Egor Babaev * \\ Institute for Theoretical Physics, Uppsala University, Box 803, 75108 Uppsala, Sweden \\ NORDITA, Blegdamsvej 17, DK-2100 Copenhagen, Denmark \\ Science Institute, University of Iceland, Dunhaga 3, 107 Reykjavik, Iceland
}

\begin{abstract}
We discuss linear topological defects allowed in two-gap superconductors and equivalent extended Faddeev model. We show that in these systems there exist vortices which carry an arbitrary fraction of magnetic flux quantum. Besides that we discuss topological defects which do not carry magnetic flux and describe features of ordinary one-magnetic-flux-quantum vortices in the two-gap system. The results could be relevant for the newly discovered two-band superconductor $M g B_{2}$.
\end{abstract}

A fundamental property of the Abelian Higgs model is the quantization of magnetic flux [1]. In an ordinary superconductor the Abrikosov vortices can carry only integer number of magnetic flux quanta. The intriguing possibilities of topological defects carrying a fraction of flux quantum have long attracted interest and several nontrivial realizations were identified. For example, a half flux-quantum vortex in a spin- 1 condensate is a configuration where a Cooper pair has its spin reversed when moving around the vortex core (this is an analogue of an Alice string in high energy physics where a particle moving around the string flips its charge or enters a "shadow world") also a half flux-quantum vortex can be formed on a junction of 3 grain boundaries in a crystal etc [2]. In this paper we discuss vortices in two-gap superconductors [3,4] (known in particle physics as a Higgs doublet model [5]) and in the extended Faddeev model. We show that these vortices can carry an arbitrary fraction of magnetic flux quantum.

Experimentally, two-gap superconductivity has been observed in the transition metals $\mathrm{Nb}, \mathrm{Ta}, \mathrm{V}$ and in $\mathrm{Nb}$ doped $\mathrm{SrTiO}_{3}$ [6]. More recent experiments indicate the two-gap nature of superconductivity in $\mathrm{MgB}_{2}$ [7] and $2 \mathrm{H}-\mathrm{NbSe}_{2}$ [8]. Two-gap models appear also in the theoretical studies of liquid metallic hydrogen, which should allow superconductivity of both electronic and protonic Cooper pairs [9]. In liquid metallic deuterium a deuteron superfluidity may be present along with superconductivity of electronic Cooper pairs [9]. Other realizations of the two-gap system are superconductors with two types of pairing (e.g. a mixture of $s$ - and $p$-wave condensates).

A two-gap superconductor is described by a twoflavour Ginzburg-Landau free energy functional:

$$
\begin{aligned}
& F=\frac{1}{2 m_{1}}\left|(\nabla+i e \mathbf{A}) \Psi_{1}\right|^{2}+\frac{1}{2 m_{2}}\left|(\nabla+i e \mathbf{A}) \Psi_{2}\right|^{2} \\
& +V\left(\left|\Psi_{1,2}\right|^{2}\right)+\eta\left[\Psi_{1}^{*} \Psi_{2}+\Psi_{2}^{*} \Psi_{1}\right]+\frac{\mathbf{B}^{2}}{2}
\end{aligned}
$$

where $\Psi_{\alpha}=\left|\Psi_{\alpha}\right| e^{i \phi_{\alpha}}$ and $V\left(\left|\Psi_{1,2}\right|^{2}\right)=-b_{\alpha}\left|\Psi_{\alpha}\right|^{2}+$
$\frac{c_{\alpha}}{2}\left|\Psi_{\alpha}\right|^{4}$ and $\eta$ is a characteristic of the interband Josephson coupling strength [1].

Many exotic properties of (11) are obscured in the Ginzburg-Landau presentation of the free energy functional. In [10] it was shown that there exists an exact equivalence mapping between the model (1) and an extended version of Faddeev's $O(3)$ nonlinear $\sigma$-model [11], which describes the two-gap superconductors in terms of gauge invariant variables which explicitly show the degrees of freedom present in the system. This model consists of a three-component unit vector $\overrightarrow{\mathbf{n}}$ in interaction with a massive vector field $\vec{C}$ and a density-related variable $\rho$ [12]:

$$
\begin{aligned}
F & =\frac{\rho^{2}}{4}(\nabla \overrightarrow{\mathbf{n}})^{2}+(\nabla \rho)^{2}+\frac{\rho^{2}}{16} \overrightarrow{\mathrm{C}}^{2}+V\left(\rho, n_{3}\right)+\rho^{2} K n_{1} \\
& +\frac{1}{32 e^{2}}\left(\partial_{i} C_{j}-\partial_{j} C_{i}-\overrightarrow{\mathbf{n}} \cdot \partial_{i} \overrightarrow{\mathbf{n}} \times \partial_{j} \overrightarrow{\mathbf{n}}\right)^{2}
\end{aligned}
$$

where $\partial_{i}=\frac{d}{d x_{i}}, V=A+B n_{3}+C n_{3}^{2}$. The models (1) and (2) are connected in the following way [10]: coefficients $A, B, C$ are given by: $A=\rho^{2}\left[4 c_{1} m_{1}^{2}+4 c_{2} m_{2}^{2}-b_{1} m_{1}-\right.$ $\left.b_{2} m_{2}\right] ; \quad B=\rho^{2}\left[8 c_{2} m_{2}^{2}-8 c_{1} m_{1}^{2}-b_{2} m_{2}+b_{1} m_{1}\right] ; \quad C=$ $4 \rho^{2}\left[c_{1} m_{1}^{2}+c_{2} m_{2}^{2}\right]$. The position of the unit vector $\overrightarrow{\mathbf{n}}$ on the sphere $S^{2}$ can be characterized by two angles as follows: $\overrightarrow{\mathbf{n}}=(\sin \theta \cos \gamma, \sin \theta \sin \gamma, \cos \theta)$, where $\gamma=\left(\phi_{1}-\right.$ $\left.\phi_{2}\right) ;\left|\Psi_{1,2}\right|=\left[\sqrt{2 m_{1}} \rho \sin \left(\frac{\theta}{2}\right), \sqrt{2 m_{2}} \rho \cos \left(\frac{\theta}{2}\right)\right] ; \overrightarrow{\mathrm{C}}=$ $\frac{i}{m_{1} \rho^{2}}\left\{\Psi_{1}^{*} \nabla \Psi_{1}-\Psi_{1} \nabla \Psi_{1}^{*}\right\}+\frac{i}{m_{2} \rho^{2}}\left\{\Psi_{2}^{*} \nabla \Psi_{2}-\Psi_{2} \nabla \Psi_{2}^{*}\right\}-$ $\frac{2 e}{\rho^{2}}\left(\frac{\left|\Psi_{1}\right|^{2}}{m_{1}}+\frac{\left|\Psi_{2}\right|^{2}}{m_{2}}\right)$ A. We consider the system in the presence of an interband Josephson coupling $\eta\left[\Psi_{1}^{*} \Psi_{2}+\right.$ $\left.\Psi_{2}^{*} \Psi_{1}\right]=\rho^{2} K n_{1}$ where $K \equiv 2 \eta \sqrt{m_{1} m_{2}}$. The potential term $V$ in (2) determines the energetically preferred ground state value for $n_{3}$, which corresponds to uniform density of both condensates. We denote it as $\tilde{n}_{3} \equiv\left[\frac{N_{2}}{m_{2}}-\frac{N_{1}}{m_{1}}\right]\left[\frac{N_{1}}{m_{1}}+\frac{N_{2}}{m_{2}}\right]^{-1}$ where $N_{1,2}$ stands for the

*email: egor@nordita.dk http://www.teorfys.uu.se/PEOPLE/egor/ 
average $<\left|\Psi_{1,2}\right|^{2}>$. Thus, in the presence of the intrinsic Josephson effect, the ground state value of $\overrightarrow{\mathbf{n}}$ corresponds to a point where $n_{1}=-1$ on a circle specified by the condition $n_{3}=\tilde{n}_{3} \equiv \cos \tilde{\theta}$. We note that (2) shows the Meissner effect, which is manifest in generation of the mass for $\vec{C}[10]$. The corresponding length scale $\lambda$ is the London magnetic field penetration length: $\lambda^{2}=\frac{1}{e^{2}}\left[\frac{\left|\Psi_{1}\right|^{2}}{m_{1}}+\frac{\left|\Psi_{2}\right|^{2}}{m_{2}}\right]^{-1}$. One can observe from (2) that along with the Meissner effect in a two-band superconductor there exists a neutral boson. That is, if one considers a uniform density of condensates, the vector field $\vec{C}$ decouples from the field $\overrightarrow{\mathbf{n}}$, because $\overrightarrow{\mathbf{n}} \cdot \partial_{i} \overrightarrow{\mathbf{n}} \times \partial_{j} \overrightarrow{\mathbf{n}} \propto$ $\sin \theta\left[\partial_{i} \gamma \partial_{j} \theta-\partial_{i} \gamma \partial_{j} \theta\right]=0$, when $n_{3}=\cos \tilde{\theta}=$ const. Thus, when $\eta=0$, the system possesses a massless neutral $O(2)$ excitation associated with the phase variable $\gamma=\left(\phi_{1}-\phi_{2}\right)$. This phenomenon has no counterpart in one-gap superconductors where the Goldstone boson associated with $O(2)$ symmetry is "eaten" by the Meissner effect. In the presence of the Josephson effect $(\eta \neq 0)$ the variables still decouple but the Josephson term $\rho^{2} K n_{1}$ breaks the neutral $O(2)$ symmetry by giving a nonzero mass to $n_{1}$.

Let us discuss vortices in the system (2). In the London limit $\left(\left|\Psi_{i}\right|=\right.$ const $)$, the eqs. (11), (2) become:

$$
\begin{aligned}
& F=\frac{\rho^{2}}{4}(\nabla \overrightarrow{\mathbf{n}})^{2}+\frac{\rho^{2}}{16} \overrightarrow{\mathrm{C}}^{2}+\frac{1}{32 e^{2}}\left[\partial_{i} \mathrm{C}_{j}-\partial_{j} \mathrm{C}_{i}\right]^{2}+\rho^{2} K n_{1}= \\
& \frac{\rho^{2}}{4} \sin ^{2} \tilde{\theta}(\nabla \gamma)^{2}+\rho^{2}\left[\sin ^{2}\left(\frac{\tilde{\theta}}{2}\right) \nabla \phi_{1}+\cos ^{2}\left(\frac{\tilde{\theta}}{2}\right) \nabla \phi_{2}-e \mathbf{A}\right]^{2} \\
& +\frac{\mathbf{B}^{2}}{2}+\rho^{2} K \sin \tilde{\theta} \cos \gamma
\end{aligned}
$$

From this expression one can observe that the vortices characterized by $\Delta\left(\phi_{1}+\phi_{2}\right) \equiv \oint_{\sigma} d l\left[\nabla\left(\phi_{1}+\phi_{2}\right)\right]=$ $4 \pi m ; \Delta\left(\phi_{1}-\phi_{2}\right)=0$, (where we integrate over a closed curve $\sigma$ around the vortex core) is the analogue of $m$-flux quanta Abrikosov vortices in an ordinary superconductor characterized by $\frac{|\Psi|^{2}}{m}=\left(\frac{\left|\Psi_{1}\right|^{2}}{m_{1}}+\frac{\left|\Psi_{2}\right|^{2}}{m_{2}}\right)$. Let us observe that if both phases $\phi_{1,2}$ change by $2 \pi$ around the core then a vortex carries one quantum of magnetic flux.

The vortices characterized by $\Delta\left(\phi_{1}-\phi_{2}\right)=4 \pi n$ in the case where $\frac{\left|\Psi_{1}\right|^{2}}{m_{1}} \neq \frac{\left|\Psi_{2}\right|^{2}}{m_{2}}$ have nontrivial structure. Let us first consider the case when $\Delta\left(\phi_{1}+\phi_{2}\right)=0$ and $\Delta\left(\phi_{1}-\phi_{2}\right)=4 \pi$. First of all such a vortex features a neutral superflow characterized by a $4 \pi$ gain in the variable $\gamma$. In the case of $\eta \neq 0$ this vortex is described by the sine-Gordon functional: $F=$ $(1 / 4) \rho^{2} \sin ^{2} \tilde{\theta}(\nabla \gamma)^{2}+\rho^{2} K \sin \tilde{\theta} \cos \gamma$. [In the limit $\frac{\left|\Psi_{1}\right|^{2}}{m_{1}}=$ $\frac{\left|\Psi_{2}\right|^{2}}{m_{2}}$, this vortex does not involve a nontrivial configuration of the field $\vec{C}$, as can be seen from (3)]. In the case $K=\eta=0$ this vortex is equivalent to a vortex in a neutral superfluid with superfluid stiffness $(1 / 2) \rho^{2} \sin ^{2} \tilde{\theta}$. As follows from (3), in the case when $\frac{\left|\Psi_{1}\right|^{2}}{m_{1}} \neq \frac{\left|\Psi_{2}\right|^{2}}{m_{2}}$, a topological defect in the neutral field associated with $\gamma$ is necessarily accompanied by a nontrivial configuration of the charged field $\overrightarrow{\mathrm{C}}$. That is, for such a topological defect the second term in (3) becomes $\rho^{2}\left[\left\{\sin ^{2}\left(\frac{\tilde{\theta}}{2}\right)-\cos ^{2}\left(\frac{\tilde{\theta}}{2}\right)\right\} \frac{\nabla\left(\phi_{1}-\phi_{2}\right)}{2}-e \mathbf{A}\right]^{2}$. This term is nonvanishing for such a vortex configuration, which means that this vortex besides neutral vorticity also carries magnetic field. Lets us calculate the magnetic flux carried by such a vortex. The supercurrent around the core of this vortex is: $\mathbf{J}=2 e \rho^{2}\left[\left\{\sin ^{2}\left(\frac{\tilde{\theta}}{2}\right)-\right.\right.$ $\left.\left.\cos ^{2}\left(\frac{\tilde{\theta}}{2}\right)\right\} \frac{\nabla\left(\phi_{1}-\phi_{2}\right)}{2}-e \mathbf{A}\right]$. Lets us now integrate this expression over a closed path $\sigma$ situated at a distance larger than $\lambda$ from the vortex core. Indeed at a distance much larger than the penetration length the supercurrent $\mathbf{J}$, or equivalently the massive field $\vec{C}$, vanishes. Thus we arrive at the following equation:

$$
\Phi=\cos \tilde{\theta} \oint_{\sigma} \frac{1}{2} \nabla\left(\phi_{1}-\phi_{2}\right) d \mathbf{l}=\cos \tilde{\theta} \Phi_{0} \equiv \tilde{n}_{3} \Phi_{0},
$$

where $\Phi=\oint_{\sigma} \mathbf{A} d \mathbf{l}$ is the magnetic flux carried by our vortex and $\Phi_{0}=2 \pi / e$ is the standard magnetic flux quantum. From (4) it follows that in our system such a vortex can carry arbitrary fraction of magnetic flux quantum since it depends on the free parameter $\cos \tilde{\theta}$ which is a measure of the relative densities of the two condensates in the system. In the general case, a vortex characterized by the following phase changes around the core $\Delta \phi_{1}=2 \pi k_{1}$ and $\Delta \phi_{2}=2 \pi k_{2}$, carries the following flux:

$$
\Phi_{\left(k_{1}, k_{2}\right)}=\left[\sin ^{2}\left(\frac{\tilde{\theta}}{2}\right) k_{1}+\cos ^{2}\left(\frac{\tilde{\theta}}{2}\right) k_{2}\right] \Phi_{0}
$$

We should emphasis that a straightforward inspection of (2) shows that this model allows neutral vortices associated with the neutral $O(2)$ boson without a nontrivial configuration of the field $\vec{C}$ for any values of $\tilde{n}_{3}$. However such solutions (e.g. vortices in the case $\tilde{n_{3}} \neq 0$ characterized by $\Delta \gamma=2 \pi ; \vec{C} \equiv 0$ ) are unphysical because these vortices do not satisfy the condition that $\phi_{i}$ change by $2 \pi k_{i}$ around the vortex core (as follows from (3)). Thus, while the original model (1) and the extended Faddeev model (2) have the same number of degrees of freedom so that the fields $\overrightarrow{\mathbf{n}}$ and $\vec{C}$ are dynamically independed by construction, the mapping incurs a constraint on topological defects in $\overrightarrow{\mathbf{n}}$ and $\overrightarrow{\mathbf{C}}$ since $\phi_{1,2}$ appear in both of them. So, in the hydrodynamic limit in (2) there is no direct coupling between the fields $\overrightarrow{\mathbf{n}}$ and $\overrightarrow{\mathbf{C}}$, however in a non-simply-connected space a topological defect in the field $\overrightarrow{\mathbf{n}}$ necessary induce a nontrivial configuration of the field $\vec{C}$. The consequence of this is the fractionalization of magnetic flux in the model (2).

So a two-band superconductor (11) and extended Faddeev model (22) allow the following linear composite topological defects:

(i) $\Delta\left(\phi_{1}-\phi_{2}\right)=4 \pi n ; \Delta\left(\phi_{1}+\phi_{2}\right)=0$ : these are the vortices which feature neutral superflow. When the 
ground state of the variable $\overrightarrow{\mathbf{n}}$ corresponds to the equator on $S^{2}$ (that is the case when $\frac{\left|\Psi_{1}\right|^{2}}{m_{1}}=\frac{\left|\Psi_{2}\right|^{2}}{m_{2}}$ or equivalently $\cos \tilde{\theta}=0)$ these vortices do not carry magnetic flux. In the case when $\cos \tilde{\theta} \neq 0$, the only physical solutions with neutral vorticity also carry a fraction of magnetic flux quantum. In the case of nonzero Josephson coupling these vortices are described by the sine-Gordon equation (3).

(ii) $\Delta \gamma=0 ; \Delta\left(\phi_{1}+\phi_{2}\right)=4 \pi k$ these are the vortices which feature circular supercurrent and no circular neutral superflow. Such vortices carry $k$ flux quanta of magnetic field. One of the physical consequences of our analysis is that we can observe that in the two-gap superconductor (1), (9), in spite of the existence of two types of Cooper pairs, a formation of two sublattices of vortices corresponding to each of the condensates in an external field is energetically forbidden. This is because the energy per unit length of noncomposite vortices is divergent in an infinite sample both in cases of zero and nonzero Josephson coupling (in case of finite $\eta$ a vortex creates a domain wall which makes its energy per unit length divergent in infinite sample [1, 14]). We can also observe that a superconductor made up of one condensate which is of type-II and another condensate which is of type-I will in general, in the presense of an external magnetic field, form one flux quantum vortices involving both condensates and will preserve two-gap superconductivity, even if the external field exceeds the thermodynamic critical magnetic field for the type-I condensate.

(iii) $\Delta \gamma=2 \pi n ; \Delta\left(\phi_{1}+\phi_{2}\right)=4 \pi l$ : These are the vortices which may be viewed as a co-centered $l$-flux quanta Abrikosov vortex combined with a vortex with neutral vorticity with winding number $n$ carrying a fractional magnetic flux. These vortices and the vortices of type (ii) characterized by $\Delta\left(\phi_{1}+\phi_{2}\right) \geq 8 \pi$ could be unstable against decay into more simple vortices [13].

Besides composite vortices the system allows vortices where the phase of one of the condensates changes by $2 \pi$ around the core whereas the phase of the second condensate remains constant. These vortices are also principally different from ordinary Abrikosov vortices. Let us discuss these vortices in the Ginzburg-Landau formalism (1). This will provide additional perspective on the discussion and will allow us to illustrate the connection between the models (11) and (2). We shall present a solution for these vortices in the limit when the inverse mass for $n_{1}$ which is given by Josephson term is larger than the sample size. Such an approximation allows one to put $\eta$ to zero. We should note that there certainly exist systems where $\eta$ is exactly zero. That is, a model with $\eta=0$ should describe vortices in a bi-layer system: a superconductor-insulator-superconductor compound is an example of a system of two condensates coupled only by a gauge field. In such a system, a vortex in one layer carries flux through the second layer, where it also in- duces a current, which should lead to the flux fractionalization. Besides that an additional $U(1)$ symmetry appears if one considers equal-spin pairing. In that case, if the spin-orbit coupling is neglected, each spin population has its own phase, without Josephson coupling [15].

Let us recall the GL equation for the supercurrent in standard notations: $\mathbf{J}=\frac{i e}{2 m_{1}}\left[\Psi_{1}^{*} \nabla \Psi_{1}-\Psi_{1} \nabla \Psi_{1}^{*}\right]+$ $\frac{i e}{2 m_{2}}\left[\Psi_{2}^{*} \nabla \Psi_{2}-\Psi_{2} \nabla \Psi_{2}^{*}\right]+e^{2} \mathbf{A}\left[\frac{\left|\Psi_{1}\right|^{2}}{m_{1}}+\frac{\left|\Psi_{2}\right|^{2}}{m_{2}}\right]$. In the situation when only the phase $\phi_{1}$ changes by $2 \pi$ around the core while the phase of the second condensate remains constant, we have the following expression for the vector potential: $\mathbf{A}=\mathbf{J}\left[e^{2}\left(\frac{\left|\Psi_{1}\right|^{2}}{m_{1}}+\frac{\left|\Psi_{2}\right|^{2}}{m_{2}}\right)\right]^{-1}+$ $\frac{1}{e} \frac{\left|\Psi_{1}\right|^{2}}{m_{1}}\left[\frac{\left|\Psi_{1}\right|^{2}}{m_{1}}+\frac{\left|\Psi_{2}\right|^{2}}{m_{2}}\right]^{-1} \nabla \phi_{1}$. From this expression it follows that the vortex characterized by $\Delta \phi_{1}=2 \pi ; \Delta \phi_{2}=0$ carries the following fractional magnetic flux

$$
\Phi=\oint \overrightarrow{\mathbf{A}} d l=\frac{\left|\Psi_{1}\right|^{2}}{m_{1}}\left[\frac{\left|\Psi_{1}\right|^{2}}{m_{1}}+\frac{\left|\Psi_{2}\right|^{2}}{m_{2}}\right]^{-1} \Phi_{0},
$$

Let us now remark on what are the physical roots of this flux fractionalization. Further examining the solution for the vortex $\left(\Delta \phi_{1}=2 \pi, \Delta \phi_{2}=0\right)$, we can write the vector potential as $\mathbf{A}=\frac{\mathbf{r} \times \mathbf{e}_{z}}{|r|}|\mathbf{A}(r)|$ where $r$ measures the distance from the core and $\mathbf{e}_{z}$ is a unit vector pointing along the core. The magnetic field is then given by $|\mathbf{B}|=\frac{1}{r} \frac{d}{d r}(r|\mathbf{A}|)$. The equation for the current $\mathbf{J}$ can then be rewritten as

$$
-\frac{d}{d r}\left[\frac{1}{r} \frac{d}{d r}(r|\mathbf{A}|)\right]+\frac{\left|\Psi_{1}\right|^{2}}{m_{1}}\left[|\mathbf{A}| e^{2}-\frac{e}{r}\right]+\frac{\left|\Psi_{2}\right|^{2}}{m_{2}}|\mathbf{A}| e^{2}=0
$$

For such a vortex, the solution for the vector potential is

$$
\begin{aligned}
|\mathbf{A}|= & \frac{\frac{\left|\Psi_{1}\right|^{2}}{m_{1}}}{\frac{\left|\Psi_{1}\right|^{2}}{m_{1}}+\frac{\left|\Psi_{2}\right|^{2}}{m_{2}}} \frac{1}{e r}- \\
& \frac{\frac{\left|\Psi_{1}\right|^{2}}{m_{1}}}{\sqrt{\frac{\left|\Psi_{1}\right|^{2}}{m_{1}}+\frac{\left|\Psi_{2}\right|^{2}}{m_{2}}}} K_{1}\left(e \sqrt{\frac{\left|\Psi_{1}\right|^{2}}{m_{1}}+\frac{\left|\Psi_{2}\right|^{2}}{m_{2}} r} r\right)
\end{aligned}
$$

Indeed the magnetic field vanishes exponentially away from the vortex core with the characteristic length scale given by the magnetic field penetration length $\lambda=\left[e \sqrt{\frac{\left|\Psi_{1}\right|^{2}}{m_{1}}+\frac{\left|\Psi_{2}\right|^{2}}{m_{2}}}\right]^{-1}: \quad|\mathbf{B}|=$ $e \frac{\left|\Psi_{1}\right|^{2}}{m_{1}} K_{0}\left(e \sqrt{\frac{\left|\Psi_{1}\right|^{2}}{m_{1}}+\frac{\left|\Psi_{2}\right|^{2}}{m_{2}} r}\right)$. Also, in contrast to the Abrikosov vortex [1], besides having the fractionalization of magnetic flux our vortex also features the neutral vorticity. This, in particular, can be seen by substituting the solution (7) into (11). Then, at length scales larger than the magnetic field penetration length from the vortex core, we have the following expression for the energy density: $F=\frac{1}{2 m_{1}}\left|\left(\nabla+i \frac{\left|\Psi_{1}\right|^{2}}{m_{1}}\left[\frac{\left|\Psi_{1}\right|^{2}}{m_{1}}+\frac{\left|\Psi_{2}\right|^{2}}{m_{2}}\right]^{-1 / 2} \frac{1}{r}\right) \Psi_{1}\right|^{2}+$ 
$\frac{1}{2 m_{2}}\left|\left(i \frac{\left|\Psi_{1}\right|^{2}}{m_{1}}\left[\frac{\left|\Psi_{1}\right|^{2}}{m_{1}}+\frac{\left|\Psi_{2}\right|^{2}}{m_{2}}\right]^{-1 / 2} \frac{1}{r}\right) \Psi_{2}\right|^{2}$. Thus the energy per unit length of the vortex $\left(\Delta \phi_{1}=2 \pi, \Delta \phi_{2}=0\right)$ is divergent. This is due to the fact that such a topological configuration necessarily induces in a two-gap system the neutral superflow. Indeed the above expression for $F$ is similar to the energy density of a vortex in a neutral system with effective stiffness $\frac{\left|\Psi_{1}\right|^{2}}{m_{1}} \frac{\left|\Psi_{2}\right|^{2}}{m_{2}}\left[\frac{\left|\Psi_{1}\right|^{2}}{m_{1}}+\frac{\left|\Psi_{2}\right|^{2}}{m_{2}}\right]^{-1}$ :

$$
F_{n}=\frac{1}{2} \frac{\left|\Psi_{1}\right|^{2}}{m_{1}} \frac{\left|\Psi_{2}\right|^{2}}{m_{2}}\left[\frac{\left|\Psi_{1}\right|^{2}}{m_{1}}+\frac{\left|\Psi_{2}\right|^{2}}{m_{2}}\right]^{-1}\left|\nabla \mathrm{e}^{i \phi_{1}}\right|^{2}
$$

This shows transparently the physical origin of the presence in the system of a massless neutral boson; a topologically nontrivial configuration $\left(\Delta \phi_{1}=2 \pi, \Delta \phi_{2}=0\right)$, besides having a current in the condensate $\Psi_{1}$ also necessarily induces current in the condensate $\Psi_{2}$. Albeit in such a configuration there are no gradients of $\phi_{2}$, however the two condensates are not independent but are connected by the vector potential. The admixture of an oppositely directed supercurrent of the condensate $\Psi_{2}$ leads to the situation when the two supercurrtents partially compensate the induced by each other magnetic field. This leads to the existence of the effective neutral superflow in the system (which in particular should dominate long-range interaction between vortices). Moreover it is the fact that the two currents partially compensate induced by each other magnetic field, which leads to the fractionalization of magnetic flux. We stress that the vortex solutions $\left(\Delta \phi_{1}=2 \pi, \Delta \phi_{2}=0\right)$ in this model, albeit being topologically stable, however can not form as an energetically preferred state in external field. In an external field the system will form the composite vortices $\left(\Delta \phi_{1}=2 \pi, \Delta \phi_{2}=2 \pi\right)$ described above.

There is however an experimental set up which should allow one to directly observe $\left(\Delta \phi_{1}=2 \pi, \Delta \phi_{2}=0\right)$ excitations. That is, in a two - gap superconducting film described by (11) there should occur thermal creation of pairs of vortices and antivortices with neutral vorticity and fractional magnetic flux. The fact that this type of vortex features both confined magnetic field and longrange interaction due to neutral superflow will allow: (i) detection of the Berezinskii-Kosterlitz-Thouless transition in a system of these vortices by standard experimental techniques like flux-noise measurements or measurements in an applied dynamic magnetic field (like in a system of Abrikosov vortices [16]). (ii) such measurements straightforwardly allow one to extract data about type of interaction between a vortex and an antivortex in the system. In spite of being by definition sensitive only to magnetic flux carrying vortices these measurements should give exactly the picture of the BKT transition like in a neutral $U(1)$ system. That is, the transition should be a pure BKT transition but not a "would be" BKT transition like in a system of Abrikosov vortices.
As it is well known there is no true BKT transition is a system of Abrikosov vortices: because their interaction is screened by the Meissner effect and a BKT transition is replaced by a crossover which is observable only when penetration length is sufficiently large [17. Moreover the BKT transition in a system of these vortices should be observable even in a type-I system (both in the limits $\eta=0$ and when $\eta$ is large, where one has sine-Gordon vortices interacting with a linear potential [13]).

In conclusion, we studied vortices in a two-gap superconductor. We have shown that the system allows several types of vortices, such as vortices carrying an arbitrary fraction of the magnetic flux quantum, which have no counterpart in ordinary one-gap superconductors or two-gap neutral superfluids. This study also has interdisciplinary interest because similar models are highly relevant in particle physics 5,18 .

It is a great pleasure to thank L.D. Faddeev, D. Gorokhov, A. J. Niemi, G. Volovik, K. Zarembo, V. Cheianov, Y.M. Cho, O.Festin, S. Girvin and P. Svedlindh for interesting discussions and/or comments. This work has been supported by grant STINT IG2001062, the Swedish Royal Academy of Science and Goran Gustafsson Stiftelse UU/KTH. The author also acknowledges NorFA mobility scholarship and Thordur Jonsson and Larus Thorlacius for hospitality during his stay at University of Iceland.

[1] A.A. Abrikosov Sov. Phys. JETP 51174 (1957)

[2] For a recent review and citations see G. E. Volovik Proc.Nat.Acad.Sci. 972431 (2000).

[3] H. Suhl, B.T. Matthias, L.R. Walker, Phys.Rev.Lett.3, 552 (1959)

[4] A.J. Leggett, Prog. of Theor.Phys. 36, 901 (1966)

[5] T.D. Lee, Phys.Rev. D8 1226, (1973)

[6] see e.g. L. Shen et. al. Phys. Rev. Lett. 141025 (1965); G. Binnig et. al. Phys. Rev. Lett. 45, 1352 (1980)

[7] see e.g. F. Bouquet et. al. Phys. Rev. Lett. 87047001 (2001) Amy Y. Liu et. al., Phys. Rev. Lett. 87087005 (2001) P. Szabo et. al. Phys. Rev. Lett. 87, 137005 (2001)

[8] T. Yokoya et. al. Science 2942518 (2001)

[9] K. Moulopoulos and N. W. Ashcroft Phys. Rev. B 59 12309 (1999); J. Oliva and N. W. Ashcroft Phys. Rev. B 301326 (1984); N. W. Ashcroft J. Phys. A129 12 (2000).

[10] E. Babaev, L.D. Faddeev, A.J. Niemi Phys. Rev. B65 100512 (2002)

[11] L. Faddeev, Quantisation of Solitons, preprint IAS Print75-QS70, 1975; and in Relativity, Quanta and Cosmology vol. 1, M. Pantaleo, F. De Finis (eds.), Johnson Reprint, 1979

[12] Similar model has been found to be relevant for spin-triplet superconductors E. Babaev Phys. Rev. Lett. 88, 177002 (2002) 
[13] More details will be present elsewhere. See also E. Babaev cond-mat/0201547. E. Babaev and D. Gorokhov in preparation.

[14] The energy of the fractional flux vortices in a such a system will however be finite in a mesoscopic sample. Also, in a granular superconductor, lattice effects may rise mass of the neutral $O(2)$ boson which will make the energy per unit length of this defect finite.

[15] The author thanks G.E. Volovik for pointing out this circumstance.

[16] P. Minnhagen, Rev. Mod. Phys. 59, 1001 (1987)

[17] We should also stress that, as it was shown by Pearl, in a one-gap superconducting film, vortices also interact due to existence of a "mushroom-like" mag- netic field outside film surface. This effect gives the vortices an $1 / r$ interaction at a distance larger than [penetration length $]^{2} /[$ film thickness $]$ from the vortex core, while at a distance shorter than that the vortices interact logarithmically: J. Pearl, Appl. Phys. Lett. 5, 65 (1964); and in Low Temperature Physics-LT 9, edited by J.G. Daunt et al. (Plenum, New York, 1965), p. 566. In contrast, the fractional flux vortices considered by us have logarithmic interaction at all length scales.

[18] L. Faddeev, A.J. Niemi, Nature 387 (1997) 58; Phys. Rev. Lett. 82 (1999) 1624; Phys. Lett. B 525, 195 (2002) W. S. Bae , Y.M. Cho, S. W. Kimm Phys. Rev. D 65, 025005 (2002) Y. M. Cho, H. W. Lee, D. G. Pak Phys. Lett. B 525, 347 (2002) 